\title{
BMJ Open Comparative efficacy and safety of probiotics for the treatment of irritable bowel syndrome: a systematic review and network meta-analysis protocol
}

\author{
Man Yang, ${ }^{1}$ Yuanyuan $Y u,{ }^{2}$ Ping-Guang Lei, ${ }^{1}$ Jinqiu Yuan ${ }^{3,4}$
}

To cite: Yang M, Yu Y, Lei P-G, et al. Comparative efficacy and safety of probiotics for the treatment of irritable bowel syndrome: a systematic review and network metaanalysis protocol. BMJ Open 2019;9:e027376. doi:10.1136/ bmjopen-2018-027376

- Prepublication history for this paper is available online. To view these files, please visit the journal online (http://dx.doi org/10.1136bmjopen-2018027376).

P-GL and JY contributed equally.

Received 19 0ctober 2018 Revised 20 September 2019 Accepted 26 September 2019

A Check for updates

(C) Author(s) (or their employer(s)) 2019. Re-use permitted under CC BY-NC. No commercial re-use. See rights and permissions. Published by BMJ.

For numbered affiliations see end of article.

Correspondence to Dr Jinqiu Yuan; yuanjinqiu06@gmail.com

Professor Ping-Guang Lei; 18002575273@163.com

\section{ABSTRACT}

Introduction Irritable bowel syndrome (IBS) is a common chronic functional gastrointestinal disorder affecting approximately $10 \%$ to $25 \%$ of the adult population. A large number of clinical trials have been conducted to evaluate the efficacy of probiotics for IBS but the results were inconsistent. Previous meta-analyses have shown that probiotics are effective for IBS, but the comparative efficacy of individual species is unclear. In addition, evidence regarding the superiority of combination over single probiotic is still lacking. We, therefore, perform this study to evaluate the comparative efficacy and safety of various species of probiotics, and combination regimens for the treatment of IBS.

Methods and analysis This study is a systematic review with network meta-analysis. We will search PubMed, Scopus, The Cochrane Central Register of Controlled Trials and CINAHL for randomised controlled trials comparing probiotics with placebo or comparing different probiotics for IBS, with no language restrictions. The primary outcomes will be treatment response and global IBSsymptom score. We will initially combine included studies with traditional pairwise meta-analysis and then with random-effects network meta-analysis. We will quantify the effect of potential effect modifiers by meta-regression if appropriate. We will check the consistency assumption by testing the absolute difference between direct and indirect estimates for comparisons in closed loops. The quality of evidence will be evaluated according to the GRADE framework.

Ethics and dissemination Ethical approval is not required for literature-based studies. We will disseminate the findings through publications in peer-reviewed journals and relevant conferences.

PROSPERO registration number

CRD42018102101

\section{BACKGROUND}

Irritable bowel syndrome (IBS) is a chronic functional gastrointestinal disorder characterised by abdominal pain and altered bowel habits without any structural abnormalities. IBS affected approximately $10 \%$ to $25 \%$ of the adult population worldwide. ${ }^{1}$ In 2004 , the cost associated with IBS in the USA was over $\$ 1$ billion. ${ }^{2}$ Because IBS presents as a complex

\section{Strengths and limitations of this study}

- This network meta-analysis will quantitatively evaluate the comparative efficacy and safety of various species of probiotics, and combination regimens for the treatment of irritablebowel syndrome.

- The study will be strictly carried out according to the recommendation of Cochrane handbook for systematic reviews of interventions.

- We anticipate that the pooled effects may be influenced by high statistical heterogeneity.

- Most estimated effects among probiotics will be based on indirect comparisons, which are often considered as low quality evidence.

- The quality of original trials will affect the quality of the pooled effects.

of symptoms, the treatment needs to focus on the most bothersome symptoms and there is no standard treatment. ${ }^{3}$ Current treatments for IBS primarily include diet/lifestyle modification and pharmacological therapy. ${ }^{3}$ Pharmacological therapy is often recommended as an adjunctive treatment for patients who failed to respond to diet/lifestyle modification and for those with moderate-to-severe symptoms. Though many drug therapies, such as antispasmodics and antidepressants, showed modest efficacy, ${ }^{4}$ their clinical use is limited due to adverse events.

Recent research has shown that disturbed gut microbiota may promote the development and maintenance of IBS symptoms. ${ }^{56}$ The composition of gut microbiota differs among specific subgroups of IBS patients and healthy individuals. ${ }^{5}$ These findings promoted the research on probiotics, live or attenuated microorganisms that may have beneficial effects in humans, for the treatment of IBS. ${ }^{7}$ A large number of clinical trials have been performed to evaluate the efficacy of probiotics for IBS but have shown inconsistent results due to variations in study design, 
species and dose of probiotics. In 2014, a meta-analysis of 35 trials showed that probiotics are effective treatments for IBS, but which individual species are the most beneficial were unclear. ${ }^{8}$ The recent update of this systematic review still found no clear evidence of the effect of particular combination, species of probiotics. ${ }^{9}$

Probiotics are widely used in clinical practice despite the lack of robust evidence. ${ }^{10} \mathrm{~A}$ challenge to clinicians is to prescribe the optimal probiotic since many probiotics are available while their comparative efficacy is indeterminate. Most clinical trials investigating probiotics for IBS are placebo-controlled trials and there are hardly any studies directly comparing difference in probiotics species. In addition, whether a combination of multiple probiotics is superior to single probiotics is still unclear. Furthermore, contrary to the safe usage of probiotics for years, recent research also pay attention to their safety, particularly for immunocompromised patients, critically ill patients and cancer patients. ${ }^{11}$ The safety of probiotics for IBS also require further investigation. These questions are unlikely to be answered by one single clinical trial or traditional meta-analysis.

Network meta-analysis, in the context of a systematic review, is an extension to traditional pairwise metaanalyses in which multiple treatments are compared using both direct comparisons within trials and indirect comparisons across trials based on common comparators. ${ }^{12}{ }^{13}$ Network meta-analysis is particularly appropriate to investigate the comparative effect of multiple treatments when direct comparisons are lacking. In addition, many randomised controlled trials have been published recently but were not included in these meta-analyses. It is necessary to update the meta-anlaysis and provide the lastest evidence for clinical practice. Therefore we intend to perform this study to evaluate the comparative efficacy and safety of various species of probiotics, and combination regimens for the treatment of IBS in adults.

\section{METHODS}

\section{Study design}

This study is a systematic review with Bayesian network meta-analysis. We will perform this systematic review according to the Cochrane handbook for systematic reviews of interventions. ${ }^{14}$ We will report the final results according to the recommendation from The Preferred Reporting Items for Systematic Reviews and MetaAnalyses Extension Statement for Reporting of Systematic Reviews Incorporating Network Meta-analyses. ${ }^{15}$ We registered this study in PROSPERO international prospective register of systematic reviews on July 2018. ${ }^{16}$ This protocol was drafted according to the Preferred Reporting Items for Systematic Reviews and Meta-Analyses Protocols (PRISMA-P). ${ }^{17}$

\section{Inclusion and exclusion criteria}

We will include studies which fulfil the following criteria.
1. Types of study to be included: Randomised controlled trials

2. Participants/population: We will include trials that recruited IBS patients who had been diagnosed based on specific diagnostic criteria (the Manning criteria, Rome I criteria, Rome II criteria, Rome III criteria, Rome IV criteria or the Kruis Score).

3. Interventions: Single probiotics or a mixture of species, of any dosage regimen and any route of administration.

4. Comparators/control: Placebo, no treatment or comparison with another type of probiotic therapy.

5. Outcomes: The primary outcomes will include treatment response regarding relief of IBS symptoms and changes from baseline in global IBS-symptom score. The secondary outcomes will include changes from baseline in individual IBS-symptom score (abdominal pain, bloating, flatulence, distension and urgency), the total number of adverse events and withdrawals due to adverse events. Currently, many different standards are used for the assessment of IBS symptoms clinical trials. There will be no limitation on the IBS symptoms assessment standard in this systematic review. We will exclude studies that included IBS patients under 18 years old. Because IBS is a chronic condition and treatment often require long time to take effect, we will exclude studies with a treatment duration of $<1$ week.

\section{Search strategy and selection criteria}

We will search PubMed, Scopus, The Cochrane Central Register of Controlled Trials (CENTRAL, in The Cochrane Library) and CINAHL for potentially eligible studies. The search strategy included terms for IBS, probiotics, outcomes and randomised controlled trials (see the PubMed strategy in Box 1). The search will be limited from their inception to July 2018, with no restriction in publication status or language.

We will also search the WHO International Clinical Trials Registry Platform (apps.who.int/trialsearch/), and ClinicalTrials.gov (www.clinicaltrials.gov) for ongoing studies. Reference lists of the included studies and related review articles will be manually checked to identify additional studies.

\section{Study selection and data extraction}

Two authors will independently undertake study selection according to the predefined inclusion and exclusion criteria. Any disagreements will be resolved by discussion. All the search citations will be initially imported into reference management software and the duplicated citations will be removed automatically. We will progressively evaluate the eligibility of the remaining studies by examining titles, abstracts and full texts. The data extracted will be as follows:

1. Study information (authors, location, title and publication time); 


\section{Box 1 Search strategy for PubMed}
1. exp colonic diseases/
2. exp irritable bowel syndrome/
3. exp colonic diseases, functional/
4. irritable bowel.tw.
5. irritable colon.tw.
6. spastic colon.tw.
7. functional bowel disease ${ }^{\star}$.tw.
8. functional colonic disease ${ }^{\star}$.tw.
9. or 1 to 8
10. Probiotics/
11. probiotic $\$$.tw.
12. Lactobacill\$.tw.
13. exp Lactobacillus/
14. Bifidobacter\$.tw.
15. Bifidobacterium/
16. Enterococc\$.tw.
17. exp Enterococcus/
18. exp Escherichia/
19. Streptococcus thermophilus.tw.
20. Streptococcus thermophilus/
21. exp Saccharomyces/
22. Saccharomyces.tw.
23. or 10 to 23
24. randomised controlled trial.pt.
25. controlled clinical trial.pt.
26. randomised.ab.
27. placebo.ab.
28. clinical trials as topic/
29. randomly.ab.
30. trial.ti.
31. or 25 to 31
32. 8 and 24 and 32

2. Patient characteristics (number of patients, average age, number of females/males, race, IBS diagnosis criteria, baseline IBS severity and IBS duration);

3. Intervention and control (the species of probiotics, dosage, frequency, route of administration, duration of treatment);

4. Methodological information (description for randomisation, sequence concealment, blinding, incomplete outcome data, selective reporting and other potential risk of bias);

\section{Outcomes.}

\section{Risk of bias (quality) assessment}

The risk of bias for included studies will be evaluated using the Cochrane Collaboration's tool for assessing risk of bias. ${ }^{18}$ For each domain, we will categorise the risk of bias as low, unclear or high risk of bias. The domains for risk of bias are as follows:

1. Selection bias (randomisation sequence generation and allocation concealment);

2. Performance bias (blinding of participants and personnel);

3. Detection bias (blinding of outcome assessment);

4. Attrition bias (incomplete outcome data).

5. Reporting bias (selective reporting);
6. Other bias (including baseline imbalance, claimed to have been fraudulent, differential diagnostic activity and contamination).

\section{Data analysis}

We will initially analyse the effect by traditional pairwise meta-analysis. We will pool the effect by individual probiotic species, or combination regimens. We will use OR as the effect measure for dichotomous outcomes as OR is mathematically superior to risk ratios and easier to be modelled in network meta-analyses. ${ }^{19}{ }^{20}$ We will use standard mean difference for continuous outcomes because various scales are expected to be applied in included trials. Heterogeneity among studies will be assessed with the Q-test and the $\mathrm{I}^{2}$ index statistic. The effect will be pooled with a random-effects model accounting for potential between-study heterogeneity. We will evaluate publication bias using funnel plots if eight or more studies are involved in the meta-analysis. The funnel plot asymmetry will be evaluated by Egger's test.

We will then pool all studies using a random-effects network meta-analysis model within a Bayesian framework since random-effect model often give a more conservative result and our previous experience suggest random-effect models generally have a better goodness of fit. ${ }^{21} 22$ The network meta-analysis models will take placebo as reference because previous meta-analyses have shown that placebo was used as the control in most clinical trials. ${ }^{89}$ We will evaluate the transitivity assumption by comparing the distribution of clinical and methodological variables that could act as effect modifiers across treatment comparisons. ${ }^{23}{ }^{24}$ We will quantify the effects by meta-regression analysis through adding covariates (mean age, the proportion of females, treatment duration, the proportion of IBS subtype and risk of bias) to the network meta-analysis models if appropriate. We will check the consistency by testing the absolute difference between direct and indirect estimates for one of the comparisons in the closed loops. ${ }^{25}$ We will evaluate the mean ranks and use two-dimensional ranking plots to demonstrate the relative efficacy and safety. The quality of evidence for the estimates of the primary outcomes will be evaluated with the GRADE approach for network meta-analysis. ${ }^{26}$

We will perform sensitivity analyses to assess robustness of results according to sample size (excluding small studies with sample size $<50$ ) and study quality (excluding studies with high risk in one or more domains, or with unclear risk in three or more domains by the Cochrane Collaboration's tool for assessing risk of bias) on primary outcomes. Data will be analysed using Stata V.12 (StataCorp, College Station, Texas) and WinBUGS V.1.4.

\section{Patient and public involvement}

There was no patient or public involvement in the preparation of this protocol. 


\section{DISCUSSION}

Despite the lack of robust evidence, probiotics are widely used for IBS in clinical practice. Prescribing the optimal probiotics has been difficult for clinicians due to the lack of comparative effect research. In this systematic review and network meta-analysis, we will evaluate the comparative efficacy and safety of various species of probiotics and combination regimens for the treatment of IBS in adults. As comparisons of probiotics have been rarely directly evaluated by randomised trials, this study will provide the current best evidence.

We anticipate several limitations in this study. First, the pooled effects may be influenced by high statistical heterogeneity. ${ }^{89}$ The source of heterogeneity may include clinical characteristics of participants (as age, sex and IBS subtype) and study methodologies. There will be a chance for us to adjust these factors by adding covariates to the network meta-analysis models. Second, we anticipate that most estimated effects among probiotics will be based on indirect comparisons, for which the quality of evidence is low. Last, the quality of original trials will affect the quality of the pooled effects. To demonstrate these effects on our estimates and facilitate the use of evidence in practice, we will evaluate the quality of evidence for the primary outcomes.

\section{Author affiliations}

${ }^{1}$ Department of Gastroenterology, Songgang People's Hospital, The Second Hospital Group of Baoan, Shenzhen, China

${ }^{2}$ Department of Surgery, Prince of Wales Hospital, New Territories, Hong Kong ${ }^{3}$ Scientific Research Center, The Seventh Affiliated Hospital, Sun Yat-sen University, Shenzhen, Guangdong, China

${ }^{4} \mathrm{JC}$ School of Public Health and Primary Care, The Chinese University of Hong Kong, Hong Kong, Hong Kong, China

Contributors JQY and PGL contributed to the conceptualisation of the review. MY and JQY drafted the manuscript. JQY developed the search strategy. MY, YY, JQY and PGL revised and edited the manuscript draft and search strategy. All authors approved the manuscript.

Funding This study is funded by the Funds for Key Medical Specialties in Baoan, Shenzhen (BA082018). Start-up fund for the 100 Top Talents Program, SYSU (392012).

Competing interests None declared.

Patient consent for publication Not required.

Provenance and peer review Not commissioned; externally peer reviewed.

Open access This is an open access article distributed in accordance with the Creative Commons Attribution Non Commercial (CC BY-NC 4.0) license, which permits others to distribute, remix, adapt, build upon this work non-commercially, and license their derivative works on different terms, provided the original work is properly cited, appropriate credit is given, any changes made indicated, and the use is non-commercial. See: http://creativecommons.org/licenses/by-nc/4.0/.

\section{REFERENCES}

1 Canavan C, West J, Card T. The epidemiology of irritable bowel syndrome. Clin Epidemiol 2014;6:71-80.

2 Everhart JE, Ruhl CE. Burden of digestive diseases in the United States Part I: overall and upper gastrointestinal diseases. Gastroenterology 2009;136:376-86.
3 Ford AC, Lacy BE, Talley NJ. Irritable bowel syndrome.. N Engl J Med 2017;376:2566-78.

4 Ruepert L, Quartero AO, de Wit NJ, et al. Bulking agents, antispasmodics and antidepressants for the treatment of irritable bowel syndrome. Cochrane Database Syst Rev 2011.

5 Distrutti E, Monaldi L, Ricci P, et al. Gut microbiota role in irritable bowel syndrome: new therapeutic strategies. World J Gastroenterol 2016;22:2219-41.

6 Dupont HL. Review article: evidence for the role of gut microbiota in irritable bowel syndrome and its potential influence on therapeutic targets. Aliment Pharmacol Ther 2014;39:1033-42.

7 Bhattarai Y, Muniz Pedrogo DA, Kashyap PC. Irritable bowel syndrome: a gut microbiota-related disorder? Am J Physiol Gastrointest Liver Physiol 2017;312:G52-62.

8 Ford AC, Quigley EMM, Lacy BE, et al. Efficacy of prebiotics, probiotics, and synbiotics in irritable bowel syndrome and chronic idiopathic constipation: systematic review and meta-analysis. Am J Gastroenterol 2014;109:1547-61.

9 Ford AC, Harris LA, Lacy BE, et al. Systematic review with metaanalysis: the efficacy of prebiotics, probiotics, synbiotics and antibiotics in irritable bowel syndrome. Aliment Pharmacol Ther 2018;48:1044-60.

10 Williams MD, Ha CY, Ciorba MA. Probiotics as therapy in gastroenterology: a study of physician opinions and recommendations. J Clin Gastroenterol 2010;44:631-6.

11 Sotoudegan F, Daniali M, Hassani S, et al. Reappraisal of probiotics' safety in human. Food Chem Toxicol 2019;129:22-9.

12 Caldwell DM, Ades AE, Higgins JPT. Simultaneous comparison of multiple treatments: combining direct and indirect evidence. $B M J$ 2005;331:897-900.

13 Li T, Puhan MA, Vedula SS, et al. Network meta-analysis-highly attractive but more methodological research is needed. BMC Med 2011;9:79.

14 Higgins JPT, Green S. Cochrane Handbook for systematic reviews of interventions version 5.1.0. The Cochrane collaboration, 2011.

15 Hutton B, Salanti G, Caldwell DM, et al. The PRISMA extension statement for reporting of systematic reviews incorporating network meta-analyses of health care interventions: checklist and explanations. Ann Intern Med 2015;162:777-84.

16 Yang M, YY Y, Lei PG, et al. Comparative effectiveness of probiotics for the treatment of irritable bowel syndrome: a systematic review and network meta-analysis. PROSPERO 2018 CRD42018102101. Available: http://www.crd.york.ac.uk/PROSPERO/display_record. php?ID=CRD42018102101

17 Shamseer L, Moher D, Clarke M, et al. Preferred reporting items for systematic review and meta-analysis protocols (PRISMA-P) 2015: elaboration and explanation. BMJ 2015;350:97647.

18 Higgins JPT, Altman DG, Gøtzsche PC, et al. The Cochrane Collaboration's tool for assessing risk of bias in randomised trials. BMJ 2011;343:d5928.

19 Cummings $\mathrm{P}$. The relative merits of risk ratios and odds ratios. Arch Pediatr Adolesc Med 2009;163:438-45.

20 Paramsothy S, Kamm MA, Kaakoush NO, et al. Multidonor intensive faecal microbiota transplantation for active ulcerative colitis: a randomised placebo-controlled trial. The Lancet 2017;389:1218-28.

21 Yuan JQ, Tsoi KKF, Yang M, et al. Systematic review with network meta-analysis: comparative effectiveness and safety of strategies for preventing NSAID-associated gastrointestinal toxicity. Aliment Pharmacol Ther 2016;43:1262-75.

22 Yuan J, Zhang R, Yang Z, et al. Comparative effectiveness and safety of oral phosphodiesterase type 5 inhibitors for erectile dysfunction: a systematic review and network meta-analysis. Eur Urol 2013;63:902-12.

23 Dias S, Sutton AJ, Welton NJ, et al. NICE DSU technical support document 3: heterogeneity: subgroups, meta-regression, bias and bias-adjustment, 2011.

24 Song F, Loke YK, Walsh T, et al. Methodological problems in the use of indirect comparisons for evaluating healthcare interventions: survey of published systematic reviews. BMJ 2009;338:b1147.

25 Chaimani A, Higgins JPT, Mavridis D, et al. Graphical tools for network meta-analysis in STATA. PLoS One 2013;8:e76654.

26 Puhan MA, Schünemann HJ, Murad MH, et al. A GRADE Working group approach for rating the quality of treatment effect estimates from network meta-analysis. BMJ 2014;349:g5630. 